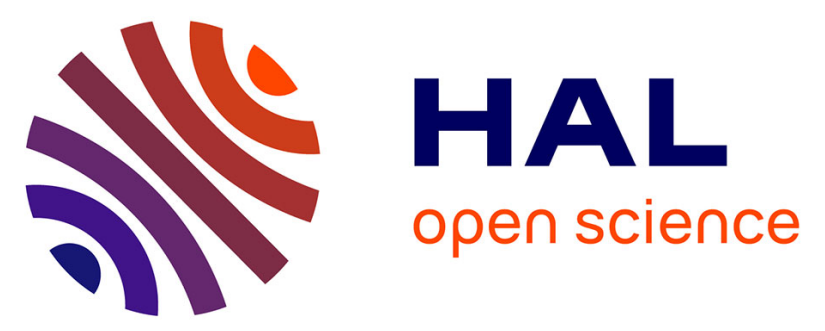

\title{
L'usage de la simulation dans les séminaires d'entreprise : entre manipulation et apprentissage de la délibération
}

\author{
Thierry Pouchol, Bernard Guéry
}

\section{To cite this version:}

Thierry Pouchol, Bernard Guéry. L'usage de la simulation dans les séminaires d'entreprise : entre manipulation et apprentissage de la délibération. RIMHE: Revue Interdisciplinaire Management, Homme(s) \& Entreprise, 2019, nº35 (2), pp.88-91. 10.3917/rimhe.035.0088 . hal-02508891

\section{HAL Id: hal-02508891 \\ https://hal.science/hal-02508891}

Submitted on 17 Mar 2020

HAL is a multi-disciplinary open access archive for the deposit and dissemination of scientific research documents, whether they are published or not. The documents may come from teaching and research institutions in France or abroad, or from public or private research centers.
L'archive ouverte pluridisciplinaire HAL, est destinée au dépôt et à la diffusion de documents scientifiques de niveau recherche, publiés ou non, émanant des établissements d'enseignement et de recherche français ou étrangers, des laboratoires publics ou privés. 


\title{
L'usage de la simulation dans les séminaires d'entreprise : entre manipulation et apprentissage de la délibération.
}

\author{
Thierry POUCHOL ${ }^{1}$ et Bernard GUERY ${ }^{2}$
}

Cet article vise à tirer des enseignements de notre expérience de consultants, concepteurs et animateurs de formation concernant l'usage de la simulation dans les séminaires d'entreprises. Différents cas sont rapportés, la simulation pouvant porter sur différents objets : on peut simuler le travail, simuler une réaction ou un sentiment, simuler le management, simuler l'innovation. La simulation peut aussi être portées par tous les acteurs des séminaires d'entreprise : le commanditaire, le prestataire, le participant.

Les quatre sens du terme «simulation » se retrouvent dans les pratiques de formation : la simulation théâtrale, la simulation sociale, la simulation par métaphore et le jeu de société. Si les travaux de Goffman $(1973,1974)$ ne peuvent qu'être évoqués dans les trois premiers, la première acception se traduit par des exercices de mimes, du chant ou la conception de saynètes. Il s'agit plus spécifiquement de mise en situation théâtrale en réponse, par exemple à la consigne des commanditaires « étonnez-nous ; amusez-nous » avec comme objectif l'appropriation des valeurs de l'entreprise. Elle place l'animateur du séminaire dans un rôle de producteur de spectacle interactif, et consiste à mettre les participants en scène, pour qu'eux-mêmes jouent un rôle. Parmi ces simulations théâtrales, il importe de mentionner le jeu de rôle à fins pédagogiques, plaçant les salariés « comme si » il était dans leur contexte professionnel, mais dans la salle de formation afin de les inviter à adopter une posture réflexive. C'est ce genre d'exercice qui est pratiqué dans les séminaires de formation à la communication, telle que la prise de parole en public, ou encore la communication interpersonnelle.

L'acception théâtrale de la simulation implique une publicité de la dualité entre la personne de l'acteur et le personnage joué. Le masque du théâtre antique manifestait ce caractère public de la différence entre l'acteur et son rôle. Au contraire, dans l'acception sociale, la simulation est une dissimulation. Par analogie, on peut évoquer les joueurs de certains sports d'équipe qui feignent la douleur dans des moments stratégiques du jeu. La simulation repose alors sur la volonté de faire croire que le sentiment ou l'émotion exprimée est réellement ressentie. Il s'agit de répondre à une attente sociale. Les techniques d'animation de groupe sollicitées lors des séminaires d'entreprise consistant, pour l'animateur, à interroger en premier les plus enthousiastes ou à instaurer un rituel d'applaudissement après chaque prestation et en fin de formation en sont une illustration : elles conduisent les participants à se soumettre à une injonction de simulation sociale. Le travail lui-même peut être simulé, les participants pouvant faire semblant de produire des résultats et « jouer» à travailler à la demande des commanditaires du séminaire d'entreprise.

\footnotetext{
${ }^{1}$ Consultant-formateur, Président du cabinet DIPSO’S - thierrypouchol@dipsos.fr

2 Enseignant-chercheur, philosophie, Institut de Philosophie Comparée (IPC) - bguery@ipc-paris.fr. Cet article est tiré d'une communication présentée au 6ème Congrès Philosophie(s) du Management à l'Institut de Management Public d'Aix-en-Provence les 4 et 5 juin 2018.
} 
Exemple de simulation sociale en séminaire d'entreprise : Il s'agit d'une entreprise de confection haut de gamme d'accessoires de mode. Un séminaire de deux jours est organisé avec l'ensemble des cadres opérationnels de l'entreprise. Cinquante personnes sont présentes, réparties en tables de huit. Une réflexion est menée, par un travail de groupe sur l'optimisation de l'organisation et des interactions des différents services entre eux. En tout, quatre séances de travail de deux heures auront lieu. Chaque séance aboutit à un livrable, sous forme de feuilles paperboard affichant les résultats de la réflexion, avec des préconisations opérationnelles. A chaque restitution par chacun des groupes, on se félicite de ces plans d'actions qui émergent, et qui feront avancer le travail en commun, on applaudit. Le séminaire se termine. Les organisateurs, la direction, la DRH, après s'être congratulés sur la qualité du travail, quittent la salle. Le lendemain, l'animateur du séminaire reçoit un coup de téléphone. Il s'agit du directeur de l'entité qui s'est réunie la veille. Il est à la recherche des feuilles de paperboards de résultats du séminaire. Il appelle pour savoir si, par hasard, ce ne serait pas l'animateur qui les aurait par devers lui. Apprenant qu'il n'en est rien, il en déduit que le personnel d'entretien les a jetées. Il conclut : " ce n'est pas grave, l'important, c'était qu'ils soient bien ensemble ».

Concernant la troisième acception, le recours à la métaphore n'est pas nouveau en sciences de gestion pour modéliser des types de management, du " manager chef d'orchestre » (Barabel et al, 2007) au " manager jardinier » (Thiébaut, 2009), ou au " manager metteur en scène » (Salgado, 2008). Dans les séminaires d'entreprise, elle correspond à une forme spécifique de simulation postulant la validité de la transposition ou du « déménagement » opéré, en référence à l'étymologie du terme "métaphore ». Ainsi, la sollicitation d'un ancien rugbyman de niveau international pour animer un séminaire de management d'équipe, ou la construction d'un séminaire sur l'éthique managériale à la lumière de l'éthique militaire ne sont pas neutres quantau choix de la métaphore sollicitée, les messages implicites qu'elles véhiculent s'avérant bien plus puissants pour modeler les esprits que les longs discours.

Enfin, les jeux de sociétés peuvent être vus comme des simulations de la réalité sociale.

Exemple de jeu de société en séminaire d'entreprise : «Un sous-traitant automobile, leader mondial dans son secteur, organise une formation pour ses managers. L'objectif est d'aider ces derniers à trouver les bonnes interactions avec les élus du personnel. La vingtaine de stagiaires, de niveau responsable d'atelier, joueront pendant quatre heures à un " jeu de l'oie "de la relation partenariale avec les instances représentatives du personnel. Avec un dé, chacun avance son pion, de case en case, pour découvrir la définition de Comité d'entreprise (CE), du Délégué du personnel (DP), du Responsable syndical (RS), ou encore approfondir les modalités du droit de grève. On trouve sur les cases des consignes comme : "vous êtes en situation de conflit, reculez de 3 cases ». Le jeu de l'oie simule le parcours d'un manager à travers les obstacles et les opportunités que représentent les interactions avec les représentants du personnel».

On retrouve dans les formations le recours à des simulations de situations de coordination, de décision ou de régulation. Parmi les exercices très classiques, le plus ancien est sans doute le « jeu de la NASA », dans lequel un groupe doit se mettre d'accord pour sélectionner un nombre restreint d'objets à emporter en expédition sur la lune. Un autre exemple consiste à construire une tour en papier sans moyens de fixation. L'objectif est de mettre en lumière les modes de régulation et de coordination dans une équipe en s'appuyant sur un apprentissage de la réflexivité en appui sur la mise en situation. La simulation se veut, dans ce cas, au service de 
l'entraînement à la délibération collective. Elle est aussi une incitation des participants à solliciter leur esprit critique, notamment dans les phases de debriefing.

Toutefois, quand elle prend la forme d'un jeu, la simulation peut être l'occasion d'une manipulation. En effet, dans la mesure où le jeu fait figure d'expression transgressive par rapport à l'ordre laborant, il peut être mobilisé pour donner l'illusion au participant d'une transgression sécurisée, et représenter un moyen de vivre la sensation de la subversion sans risque supposé pour sa carrière. La simulation peut aussi jouer le rôle d'un divertissement pour pallier le manque de sens au travail. Elle peut être enfin un moyen détourné de mobilisation au travail, en rendant ce dernier plus appétible (Marache-Francisco et Brangier, 2015).

\section{Conclusion}

A la lumière de ce retour d'expérience, la simulation peut se révéler être un moyen de manipulation, voire l'occasion de jeux de pouvoirs. Elle apparaît aussi comme un mode potentiel d'apprentissage des processus de délibération collective au travail. En fonction de son utilisation, elle peut permettre, en effet, de renforcer la coopération des acteurs organisationnels et favoriser une prise de décision collégiale.

\section{Références}

Barabel M., de Beaupuy A., Meier O., Renoult S. (2007), Portrait du manager en chef d'orchestre, Expansion Management Review, n¹26, p. 106-115.

Goffman E. (1973), 1-La présentation de soi. La mise en scène de la vie quotidienne, Paris, Les Editions de Minuit.

Goffman E. (1974), Les rites d'interaction, Paris, Les Editions de Minuit. Marache-Francisco C., Brangier E. (2015), Gamification and human-machine interaction: a synthesis, Le Travail Humain, vol. $78, \mathrm{n}^{\circ} 2$, p. 165-189.

Salgado M. (2008), Le théâtre, un outil de formation au management, Revue Française de Gestion, n 181 , p. 77-96.

Thiébaut C. (2009), La patience du manager-jardinier, Expansion Management Review, $\mathrm{n}^{\circ} 135$, p. 114-120. 